\title{
Localization of Rhythmogenic Networks Responsible for Spontaneous Bursts Induced by Strychnine and Bicuculline in the Rat Isolated Spinal Cord
}

\author{
Enrico Bracci, Laura Ballerini, and Andrea Nistri \\ Biophysics Sector and Istituto Nazionale di Fisica della Materia Unit, International School for Advanced Studies (SISSA), \\ 34013 Trieste, Italy
}

Spontaneous rhythmic bursting induced by coapplication of strychnine $(1 \mu \mathrm{M})$ and bicuculline $(20 \mu \mathrm{M})$ was observed with electrophysiological recording from pairs of lumbar ventral roots (usually L5) in an isolated preparation of the neonatal rat spinal cord. Bursting was insensitive to exogenously applied GABA or glycine, confirming that it was attributable to block of glycine and $\mathrm{GABA}_{\mathrm{A}}$ receptor-mediated inhibition. NMDA accelerated bursting in a dose-dependent manner. Complete coronal spinal transection at L3 or L6 level did not block bursting recorded from L5 or L2 roots, respectively. Gradual cutting of the cord along the midline through a sagittal plane preserved bursting activity in both disconnected sides but led to loss of synchronicity. Once the spinal cord was fully separated into left and right halves, regular bursting persisted on each side with no phase-coupling between the two preparations. Section along a frontal plane to remove dorsal horns and much of the central canal area did not affect burst frequency or left-to-right synchronicity, whereas it reduced burst duration. A quadrant preparation containing mainly a single ventral horn displayed enhanced burst frequency while bursts became very short events. Bath application of 5-hydroxytryptamine $(30 \mu \mathrm{M})$ or NMDA $(5 \mu \mathrm{M})$ increased burst frequency and decreased burst duration in all types of preparation except the isolated quadrants, in which brief bursts were accelerated but not shortened by these chemical agents. These results suggest that bursting induced by strychnine and bicuculline apparently relied on distinct mechanisms for burst triggering and intraburst structure. The first required a relatively smaller neuronal network that was confined to a ventral quadrant. Intraburst structure was dependent on a larger circuitry comprising either both ventral horns or one side of the spinal cord including more than two segments.

Key words: motoneuron; spinal cord; rhythmogenesis; inhibition; GABA; glycine
Investigating the rhythmic motor patterns of the spinal cord in vitro provides a useful tool for understanding the more complex behavior of functionally intact spinal networks. In the in vitro neonatal rat spinal cord, rhythmic patterns of motoneuronal activity can be induced by several agents, including 5-hydroxytryptamine (5-HT), NMDA, and acetylcholine (Cazalets et al., 1992; Cowley and Schmidt, 1994), which are known to modulate neuronal membrane properties (Hultborn and Kiehn, 1992). Each one of these drugs can elicit rhythmic activity consistent with locomotion (i.e., alternating bursts in flexor and extensor motoneurons on each side of the cord, referred to as fictive locomotion) (Rossignol and Dubuc, 1994), although patterns incompatible with locomotor activity (for instance, left-right alternation but in-phase activation of flexor and extensor nerves within each hindlimb) can also be manifested (Cowley and Schmidt, 1994). We have shown recently that in this preparation regular rhythmic bursts occur spontaneously in motoneurons after applying pharmacological blockers of $\mathrm{GABA}_{\mathrm{A}}$ and glycine receptors (Bracci et al., 1996). Such bursts, characterized by several intraburst oscillations, take place simultaneously in left and right ventral roots with a frequency of $\sim 2$ bursts/min and are accelerated by $5-\mathrm{HT}$ in a

\footnotetext{
Received May 10, 1996; revised July 9, 1996; accepted Aug. 9, 1996.

This work was supported by grants from the European Union (Human and Capital Mobility Programme), Telethon Foundation (Project \#823), and Istituto Nazionale di Fisica della Materia to A.N. We thank Ciba-Geigy for kindly donating CGP 52,432 and Marco Beato for his participation in some coronal section experiments.

Correspondence should be addressed to E. Bracci at SISSA, Via Beirut 4, 34013 Trieste, Italy.

Copyright (C) 1996 Society for Neuroscience $0270-6474 / 96 / 167063-14 \$ 05.00 / 0$
}

dose-dependent manner similar to that previously described for fictive locomotion (Cazalets et al., 1992). Recently, Cowley and Schmidt (1995) showed that after pharmacological block of inhibition drug-induced fictive locomotion is also converted into synchronized bursting at lower frequency. Furthermore, even organotypic cultures of the rat spinal cord display synchronized bursting in the presence of strychnine and bicuculline (Streit, 1993). These findings raise the possibility that spinal rhythmogenesis could result from cellular mechanisms that do not require synaptic inhibition, even if inhibition is certainly necessary to ensure temporal phasing of motor patterns.

The issue of localization of the rhythmogenic activity is a complex one and not resolved even for the fictive locomotor pattern. In the case of the chick embryo preparation, Ho and O'Donovan (1993) have shown that both caudal and rostral segments of the lumbosacral cord can generate this rhythmic activity and that neurons localized in the ventrolateral part of the ventral horns are sufficient for the generation of motoneuronal bursting. Moreover, they showed that during spontaneous motor episodes, two different mechanisms are responsible for burst frequency and burst duration and that these mechanisms can be affected separately by experimental lesions. This kind of investigation has not yet been performed on the mammalian spinal cord. In the rat, the localization of the locomotor network is still controversial; in fact, using Vaseline barriers applied to the surface of the rat spinal cord, Cazelets et al. (1995) have proposed that this network is localized to the upper lumbar segments, whereas other investigators suggest that the rhythmogenic neural substrate is distributed 
over a large number of segments (for discussion, see Katz, 1996). The present work investigated, in the rat spinal cord, the topography of the networks responsible for the rhythmic activity generated by simultaneous application of $\mathrm{GABA}_{\mathrm{A}}$ and glycine antagonists. To address this issue, lesions of the spinal cord were performed, including removal of segments, progressive hemisectioning, and ablation of dorsal horn tissue. Furthermore, the block of GABA or glycine receptors was functionally assessed and the sensitivity of patterned bursting to agents able to induce fictive locomotion was tested.

\section{MATERIALS AND METHODS}

Experiments were carried out on spinal cord preparations isolated from neonatal rats $(5-12 \mathrm{~d}$ old $)$ under urethane anesthesia [6 ml of $10 \%(w / v)$ solution, i.p.] as described previously (Fisher and Nistri, 1993; Ballerini et al., 1995). Spinal cords (from the mid-thoracic region to the cauda equina) were continuously superfused with oxygenated Krebs solution of the following composition (in $\mathrm{mM}$ ): $\mathrm{NaCl} 113, \mathrm{KCl} 4.5, \mathrm{MgCl}_{2} 1, \mathrm{CaCl}_{2} 2$, $\mathrm{NaH}_{2} \mathrm{PO}_{4} 1, \mathrm{NaHCO}_{3} 25$, glucose 11, pH 7.4, at $7.5 \mathrm{ml} / \mathrm{min}$. Dorsal root stimulation was performed via a miniature suction electrode using stimuli of $0.1 \mathrm{msec}$ duration, $1.5-2 \times$ threshold intensity $(2-5 \mathrm{~V})$ for monosynaptic ventral root responses, and applied once every $8 \mathrm{sec}$. For root recordings, lumbar ventral roots (predominantly L5, although L2 or L3 were also used) were connected to miniature suction electrodes filled with Krebs solution. For intracellular recording, motoneurons (functionally identified on the basis of their antidromic spike) were impaled with $3 \mathrm{M}$ $\mathrm{KCl}$-filled microelectrodes. Responses were amplified with an Axoclamp $2 \mathrm{~A}$ unit, stored on videotape for further analysis, digitized at $1-10 \mathrm{kHz}$ with the pClamp program (Axon Instruments, Foster City, CA; version 6.2), and displayed on a chart recorder. Ventral root responses were recorded with either DC-coupled (usually with $30 \mathrm{kHz}$ lowpass filter) or AC-coupled $(100 \mathrm{~Hz}$ to $10 \mathrm{kHz}$ passband) amplification. An additional 20 $\mathrm{Hz}$ lowpass filter was used occasionally. Details concerning the definition of bursts and their measurement (duration and frequency) are as reported previously (Bracci et al., 1996); cycle period was measured from the start of a burst to the beginning of the next one, and intraburst oscillation frequency was measured as $1 / t$, where $t$ is the interval between the peaks of two successive waves. Data are expressed as mean \pm SD with statistical significance evaluated by ANOVA. Drugs were applied via the bathing solution. For experiments aimed at localizing the bursting network mechanisms, lesions were performed with a small piece of razor blade after observing control responses: data were normally collected after a 15 min stabilization period after each lesion. Various types of lesion were produced: they consisted of (1) gradual cutting along the midline (sagittal plane cutting, thus yielding two hemisected preparations), (2) transverse sectioning (coronal plane cutting, thus eliminating spinal cord segments), or (3) cutting through a frontal plane yielding hemicords with ventral horns only. The latter preparation was further divided along a sagittal plane to produce a quadrant in which only one ventral horn was present. At the end of each experiment, preparations were fixed with tissue-freezing medium (Cryogel, Jung), frozen, and sectioned $(60 \mu \mathrm{m})$ with a cryostat. After staining with toluidine blue $(1 \%$ in distilled water), sections were examined with a Zeiss Axiovert 135 microscope $(5 \times$ magnification).

\section{RESULTS}

Data were collected from 37 spinal cord preparations. Previous work (Bracci et al., 1996) has shown that after application of pharmacological blockers of synaptic inhibition to the spinal cord a regular pattern of network-driven bursts appeared in motoneurons. Figure $1 A$ shows an example of bursting activity recorded intracellularly (in current-clamp conditions): bursts consisted of large amplitude depolarizations appearing at regular intervals. The average cycle period of burst episodes (measured from the start of a bursting event to the beginning of the next one) was 29 sec; average burst duration was 4.9 sec. Individual bursts were characterized by several intraburst oscillations (with frequency between 2 and $5 \mathrm{~Hz}$ ), as shown by the faster timescale trace on the right side of Figure $1 A$. When motoneuronal population activity was monitored through ventral root recording, bursts and intra- burst oscillations were also clearly detected with similar average frequency and duration (Fig. $1 B$ ). These events appeared nearly synchronously on both sides when recordings were taken from a pair of homolateral ventral roots (see examples in Figs. $3 A, 4 A$ ). This finding implies that both bursts and intraburst oscillations took place almost simultaneously in a large number of motoneurons on both sides of the cord, because even small differences in phase between motoneurons would have resulted in a less organized pattern. DC-coupled tracings from ventral roots closely resembled intracellular recordings in their slow time course (Fig. $1 B$, top row) during which clusters of action potentials were present during bursting activity (unless records were lowpassfiltered; compare expanded records in Fig. 1B). AC-coupled and/or $20 \mathrm{~Hz}$ lowpass tracings were also able to monitor bursts and intraburst oscillations (Fig. $1 C$ ) with loss of slow potential changes or fast transients. These results indicate that the main characteristics of bursting were detected with extracellular recordings (either DC- or AC-coupled) which, therefore, were much more suitable to study the effects of lesions on this preparation. Extracellular recording also allowed us to assess that bursting activity took place simultaneously in ventral (ipsi- or contralateral) roots of lumbar segments (L2-L5) of the isolated spinal cord (data not shown).

\section{Is synaptic inhibition blocked during rhythmic bursts?}

Even if strychnine and bicuculline are known to block inhibitory synaptic transmission in rat motoneurons in vitro (Wu et al., 1992), it remained to be ascertained whether in the present experimental conditions of spontaneous bursting, inhibitory synaptic processes were blocked throughout the spinal network; this was assessed by extracellular recording. In our previous study (Bracci et al., 1996), $20 \mu \mathrm{M}$ bicuculline methiodide and $1 \mu \mathrm{M}$ strychnine were used to block $\mathrm{GABA}_{\mathrm{A}}$ and glycine receptors, respectively. In the present investigation, the same concentrations of these antagonists were first tested for their ability to prevent the inhibitory action of exogenously applied glycine or GABA (in concentrations known to strongly inhibit motoneurons; Wu et al., 1992) on polysynaptic reflexes (elicited from L5 dorsal root by electrical stimulation of the homologous ipsilateral dorsal root). Dorsal root stimulations were continuously delivered at $8 \mathrm{sec}$ intervals, so as to avoid excessive depression of the reflex (Lev-Tov and Pinco, 1992). Glycine $(0.5 \mathrm{~mm})$ caused a strong reduction in reflex peak amplitude and area (Fig. $2 A$ ). On average, in three preparations such a glycine application significantly $(p<0.0001)$ decreased polysynaptic reflex peak amplitude by $37 \pm 21 \%$ and area by $64 \pm 18 \%$. These effects were readily reversed on washout (Fig. $2 A$ ). Subsequent application of $1 \mu \mathrm{M}$ strychnine caused an increase in reflex peak amplitude $(37 \pm 15 \%)$ and area $(52 \pm 25 \%)$, with the appearance of time-locked oscillations at $4-8 \mathrm{~Hz}$ (see example in Fig. $2 A$ ). In the presence of strychnine, application of $0.5 \mathrm{~mm}$ glycine failed to affect reflex amplitude, area, or oscillations. The effects of GABA application on dorsal root-evoked reflex before and after application of bicuculline were tested in the presence of $10 \mu \mathrm{M}$ CGP 52,432 so as to avoid involvement of $\mathrm{GABA}_{\mathrm{B}}$ receptors. Under these conditions $(n=3), 1 \mathrm{~mm}$ GABA significantly $(p<0.0001)$ and reversibly reduced reflex peak amplitude by $54 \pm 11 \%$ and area by $88 \pm 19 \%$ (see example in Fig. $2 B$ ). Subsequent application of $20 \mu \mathrm{M}$ bicuculline increased reflex peak amplitude by $64 \pm 13 \%$ and reflex area by $440 \pm 110 \%$ but, unlike strychnine, it did not elicit slow, rhythmic oscillations. In the presence of bicuculline, $1 \mathrm{~mm}$ GABA failed to affect reflex peak amplitude or area (Fig. 2B). 

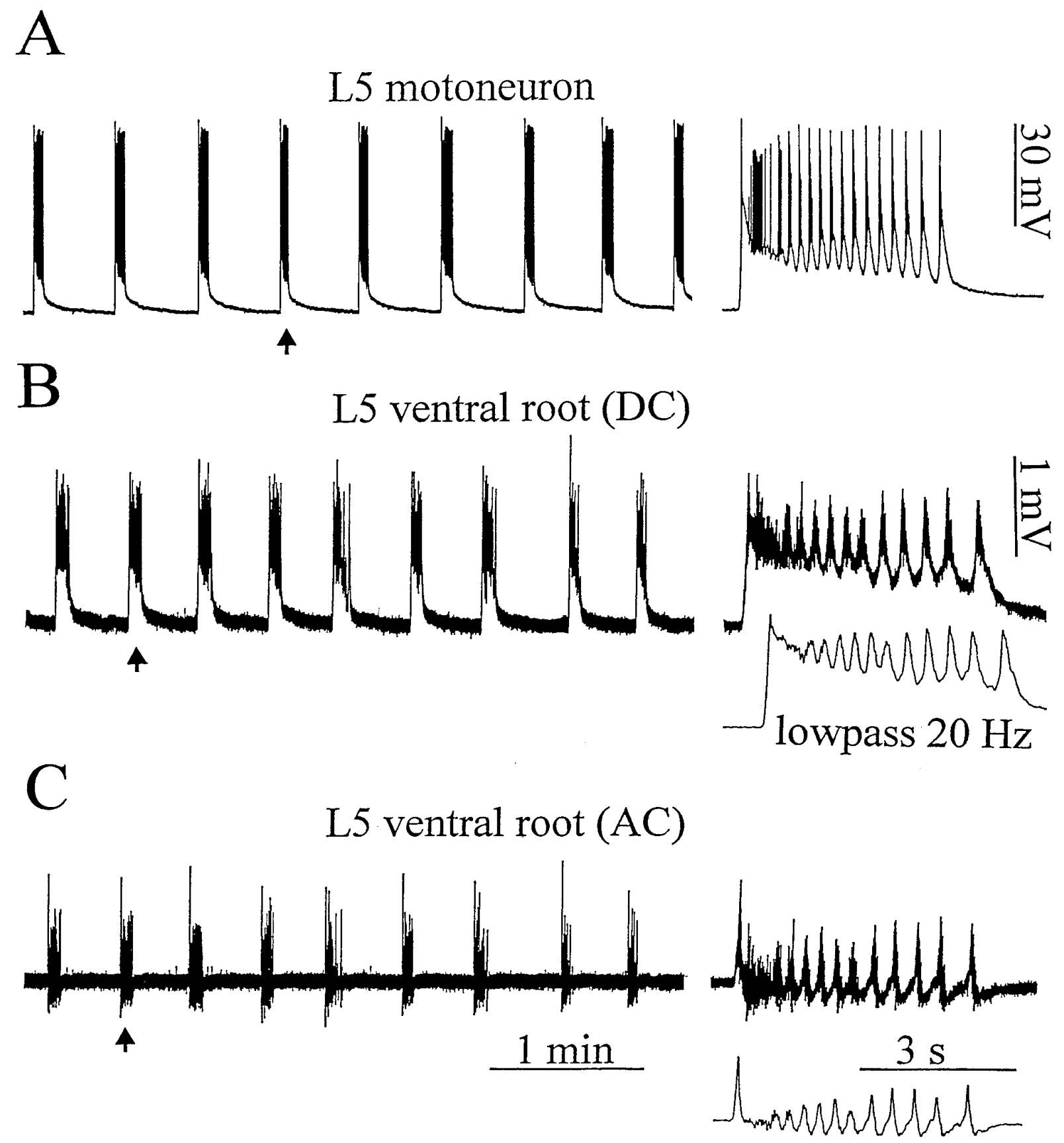

lowpass $20 \mathrm{~Hz}$

Figure 1. Comparison of intracellular and extracellular recordings of bursting activity induced by strychnine and bicuculline. $A$, Intracellular traces from L5 motoneuron (resting membrane potential $-75 \mathrm{mV}$ ) displaying regular rhythmic bursting characterized by rapid depolarization with superimposed trains of action potentials. A single event (indicated by the arrow) is shown on the right with a faster timebase to resolve intraburst oscillatory activity. $B$, DC-coupled extracellular recording of similar bursting (different preparation from $A$ ) from L5 ventral root. Note the similar bursting pattern and structure as observed with the intracellular electrode. Traces on the right represent faster timebase records [with wideband (top) or lowpass (bottom) filter] of the same individual burst indicated by the arrow. $C$, AC-coupled extracellular recording of the same activity depicted in B. Right traces show the same single event (faster timebase) with wideband (top) or lowpass (bottom) filter. Note that extracellular recording (either DC- or AC-coupled) from ventral root provides a reliable method to observe bursting activity.

After induction of spontaneous bursting by coapplication of strychnine and bicuculline to the same preparations, $0.5 \mathrm{~mm}$ glycine failed to affect burst frequency or duration (Fig. 2C; similar data were obtained on three preparations). GABA (1 mM) was equally ineffective on bursting frequency or duration in the presence of strychnine, bicuculline, and CGP 52,432 $(n=2$; Fig. $2 D)$. Prolonged application (>30 min) of CGP 52,432 (10 $\mu \mathrm{M})$ per se did not block bursting induced by strychnine and bicuculline in 4 of 4 preparations (data not shown).

Once rhythmic bursts were induced by $1 \mu \mathrm{M}$ strychnine and 20 

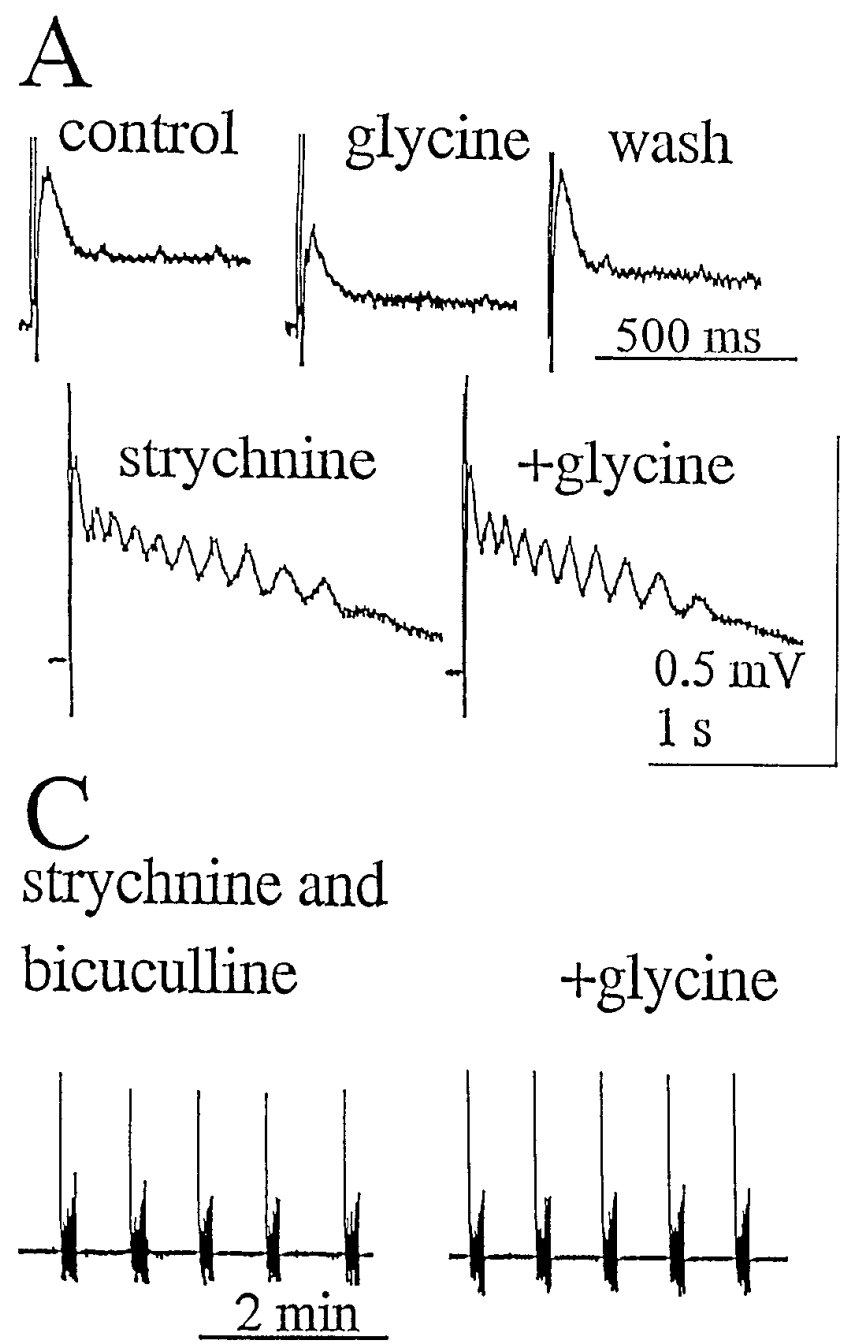

B
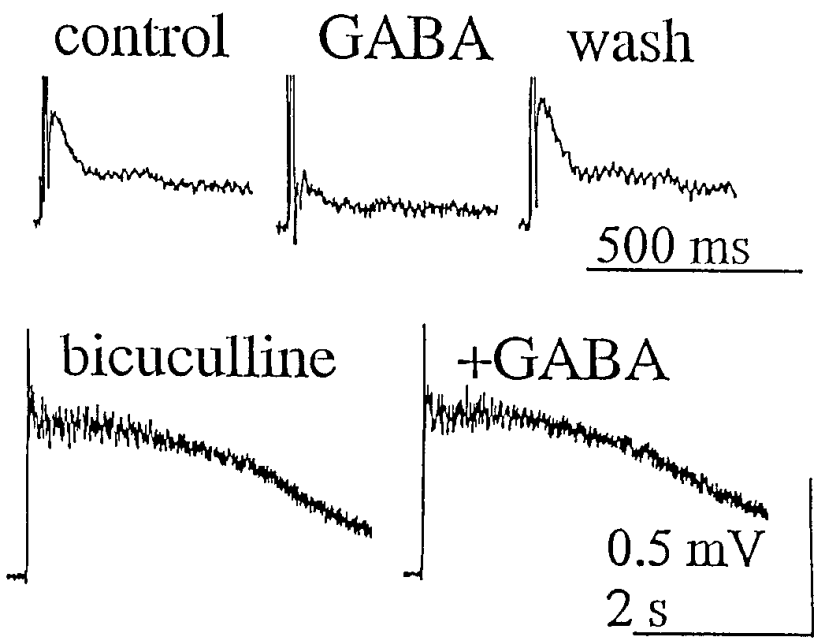

$\mathrm{D}$ strychnine and bicuculline
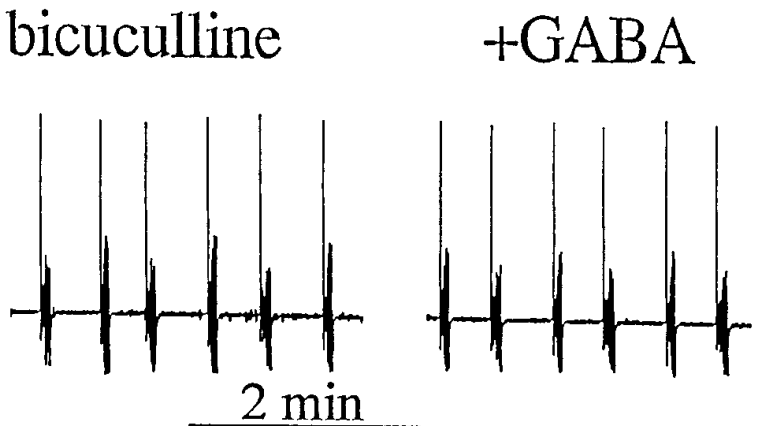

Figure 2. Effects of glycine or GABA on reflex activity and bursting. A, Top, DC records of polysynaptic reflex activity elicited by dorsal root stimulation $(0.1 \mathrm{msec}$ duration, $1 / 8 \mathrm{sec}$ frequency) before (control), during (glycine), or after (wash) application of $0.5 \mathrm{~mm}$ glycine. Responses are averaged records of 10 traces. Those in glycine solution are taken 2 min after the start of glycine superfusion; wash indicates 10 min after glycine washout. Bottom, Strychnine $(1 \mu \mathrm{M})$ enhanced reflex amplitude and induced oscillatory patterns. This effect was not sensitive to glycine ( $0.5 \mathrm{~mm}$; same preparation as above). $B$ (different preparation from $A$ ), Top, DC records of polysynaptic reflex activity elicited as described in $A$ before, during, or after application of 1 mM GABA. Bottom, Bicuculline $(20 \mu \mathrm{M})$ enhanced reflex activity and prevented the effect of GABA. All responses are averaged records of 10 traces and were recorded in the presence of CGP 52,432 $(10 \mu \mathrm{M})$ to block GABA $\mathrm{B}_{\mathrm{B}}$ receptors. $C$, AC-coupled records of spontaneous bursting activity (same preparation as in $A)$ induced by coapplication of strychnine $(1 \mu \mathrm{M})$ and bicuculline $(20 \mu \mathrm{M})$; glycine $(0.5 \mathrm{mM})$ has no depressant action, confirming that glycine receptors are effectively blocked. $D$, Comparable records (same preparation as in $B$ ) showing the lack of depressant action of GABA (1 mM) on bursting activity induced by strychnine and bicuculline (in the presence of CGP 52,432), indicating that $\mathrm{GABA}_{\mathrm{A}}$ receptors are effectively blocked.

$\mu \mathrm{M}$ bicuculline, progressive increases in strychnine concentration up to $10 \mu \mathrm{M}$ had no effect on burst frequency or duration. On the other hand, when the noncompetitive $\mathrm{GABA}_{\mathrm{A}}$ antagonist picrotoxin $(100 \mu \mathrm{M})$ was coapplied with $1 \mu \mathrm{M}$ strychnine (omitting bicuculline), rhythmic bursts similar to those elicited by bicuculline and strychnine were always observed. Under these conditions $(n=4)$, burst frequency, burst duration, and their coefficients of variation were not significantly different from the preparations treated with strychnine and bicuculline. Similarly, during the course of the experiment replacing bicuculline with picrotoxin (in the continuous presence of strychnine) did not alter the bursting pattern established with bicuculline and strychnine at the start of the recording session $(n=2)$. When coapplied with strychnine, 1 $\mathrm{mM}$ penicillin (another $\mathrm{GABA}_{\mathrm{A}}$ antagonist) was also able to induce rhythmic bursts similar to the one elicited by bicuculline and strychnine $(n=2)$.
All together, these results strongly suggest that in the rat isolated spinal cord $\mathrm{GABA}_{\mathrm{A}}$ and glycine receptors were blocked by the concentrations of strychnine and bicuculline used in the present study and that, under these conditions, rhythmogenesis should be based on mechanisms independent of these receptors. Furthermore, $\mathrm{GABA}_{\mathrm{B}}$ receptors were not necessary for rhythm generation.

\section{Sensitivity of bursting activity to NMDA and carbachol}

In our previous study (Bracci et al., 1996), the NMDA receptor antagonists CPP and APV slowed down bursting cycle period or completely suppressed bursting; in the latter case, pharmacological block of AMPA receptor desensitization reestablished bursting. It seemed interesting, therefore, to test the role of NMDA receptors more directly by applying NMDA and comparing it with another rhythmogenic substance such as carbachol (Cowley and Schmidt, 

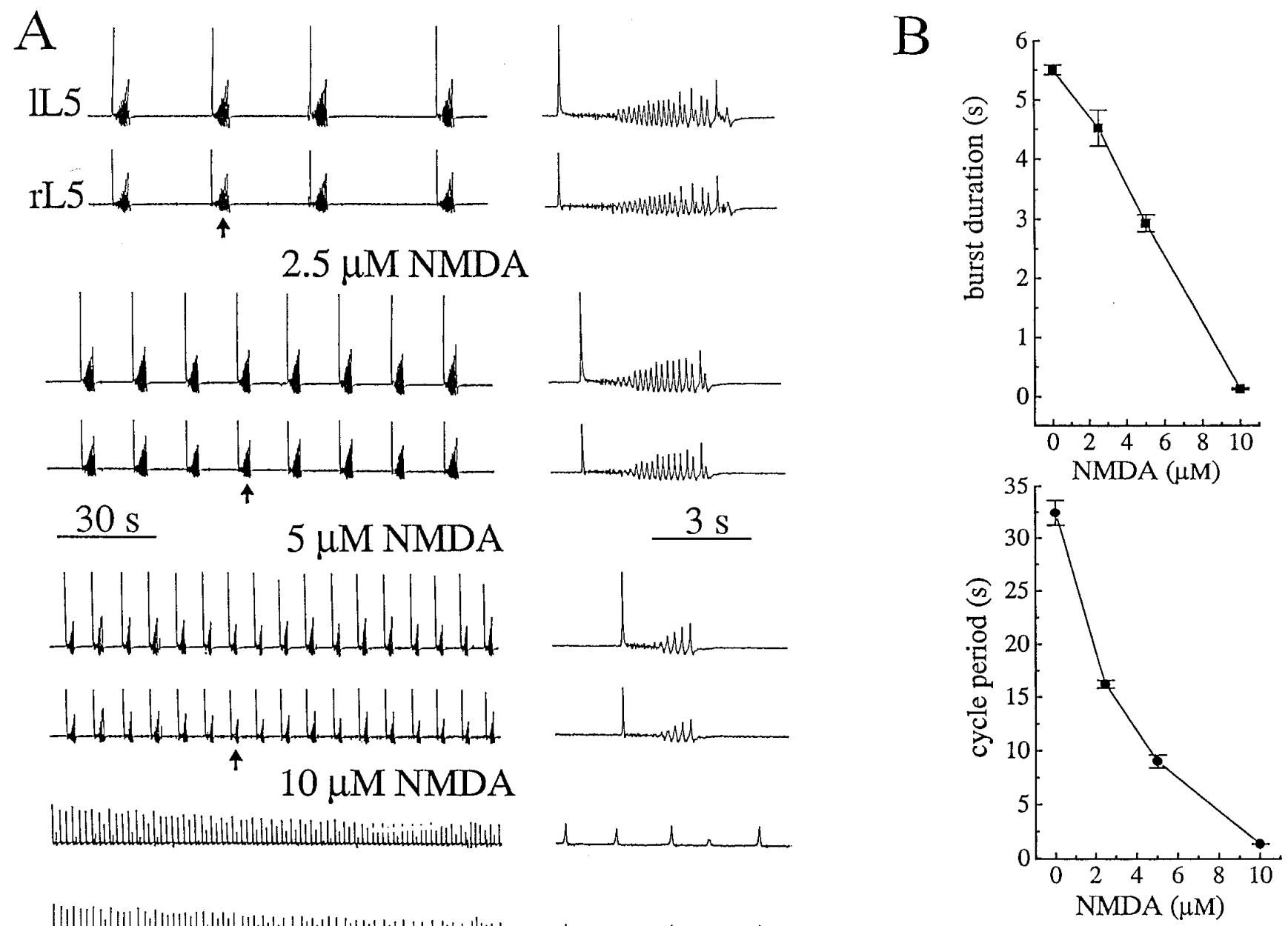

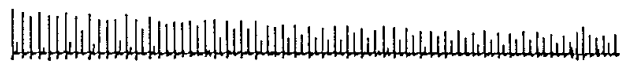

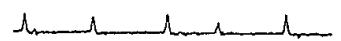

Figure 3. Dose-dependent action of NMDA on bursting activity induced by strychnine and bicuculline. $A$, Pair of AC-coupled tracings (left and right L5 ventral root; $l L 5$ and $r L 5 ; 20 \mathrm{~Hz}$ lowpass filtering) in strychnine and bicuculline solution (top) and after application of NMDA at the concentrations shown above each trace. Right panels show faster time base records of individual bursts (arrows) to depict intraburst oscillations. Note progressive acceleration of bursting activity by NMDA with concomitant decrease in burst duration. $B$, Plot of NMDA concentration versus burst duration (top) or cycle period (bottom). Each datapoint was calculated over a period of at least $5 \mathrm{~min}$ for the preparation shown in $A$.

1995). After rhythmic bursts were induced by strychnine and bicuculline, application of NMDA was able to increase burst frequency and to decrease burst duration in a dose-dependent manner. In the experiment of Figure 3, NMDA concentrations were cumulatively applied at $\sim 10 \mathrm{~min}$ intervals after rhythm stabilization. The largest NMDA dose compatible with bursting was $10 \mu \mathrm{M}$ (cycle period was $1.3 \pm 0.1 \mathrm{sec}$ and burst duration $0.13 \pm 0.02 \mathrm{sec}$; see also Fig. $3 B)$. As shown in the right panels of Figure $3 A$, intraburst oscillations were preserved with 2.5 and $5 \mu \mathrm{M}$ NMDA, even if their number progressively decreased and ultimately disappeared with $10 \mu \mathrm{M}$ NMDA, which converted bursts into single-discharge events. On a sample of four preparations, $5 \mu \mathrm{M}$ NMDA (in the presence of strychnine and bicuculline) significantly decreased cycle period (by $75 \pm 10 \%$ ) and burst duration (by $59 \pm 18 \%$ ). The cholinergic agonist carbachol (10 $\mu \mathrm{M})$ also significantly decreased cycle period (by $70 \pm 16 \%$ ) and burst duration (by $55 \pm 19 \%$ ) in the presence of strychnine and bicuculline ( $n=3$; data not shown). These data indicate that the bursting activity induced by bicuculline and strychnine was sensitive to NMDA and carbachol, two agents that are able to induce rhythmic activity in the isolated spinal cord (Cowley and Schmidt, 1995).

\section{Ablation of rostral or caudal segments}

It has been proposed recently that in the neonatal rat spinal cord the networks responsible for generation of locomotor-like activity are localized to L1/L2 lumbar segments (Cazelets et al., 1995). To investigate whether the networks localized at this level are necessary for the rhythmic activity evoked by strychnine and bicuculline, we recorded bilaterally from L5 ventral roots before and after ablation of the spinal cord segments rostral to the exit of L3 ventral roots (the lesion is schematically shown in Fig. $4 \mathrm{~A}$, left). Figure 4 shows that bursting persisted in both L5 ventral roots, and it was also maintained in the L2 ventral root (which is above the transection level; data not shown). In 6 of 6 preparations, rhythmic bursts (with intraburst oscillations) persisted at the same frequency after this lesion (compare in Fig. $4 A$, top and bottom traces, taken from the same spinal cord before and after surgical ablation). The average values for cycle period from 6 preparations confirmed the lack of change after removal of the upper spinal segments (Fig. 4B, left). Burst duration became slightly shorter, as indicated by the right records at a faster time sweep (Fig. 4A). This phenomenon, however, was not statistically significant, as 
A
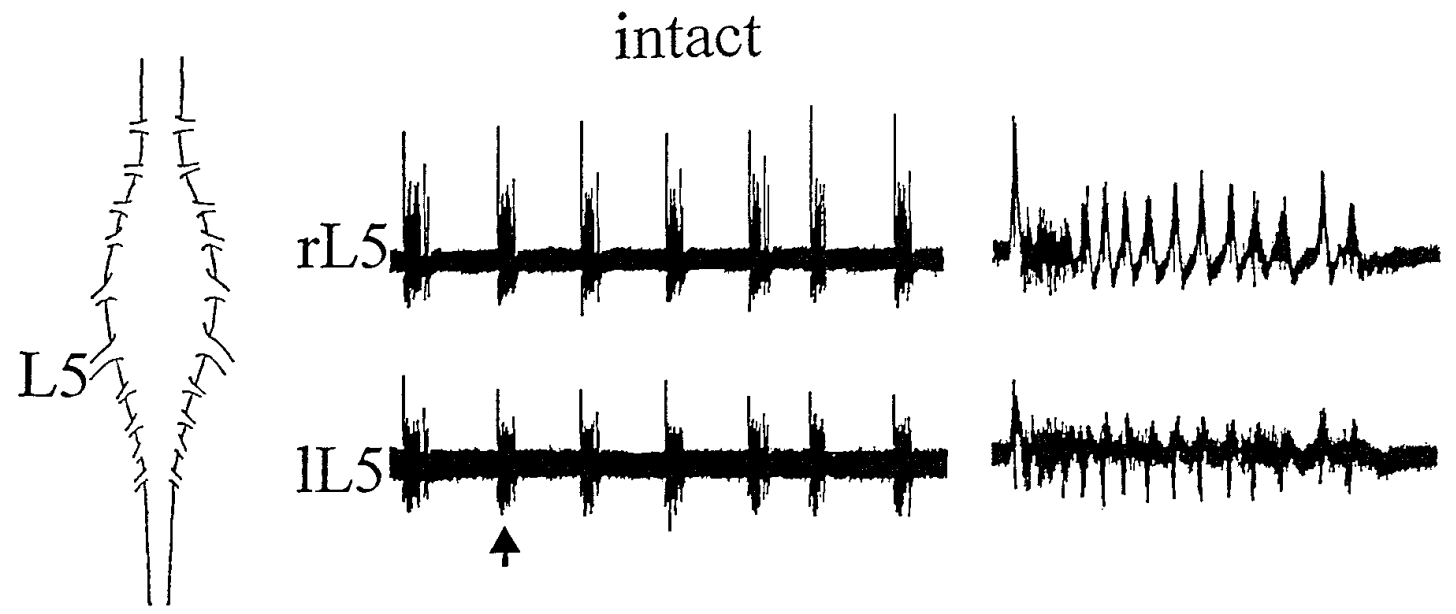

ablation rostral to $\mathrm{L} 3$
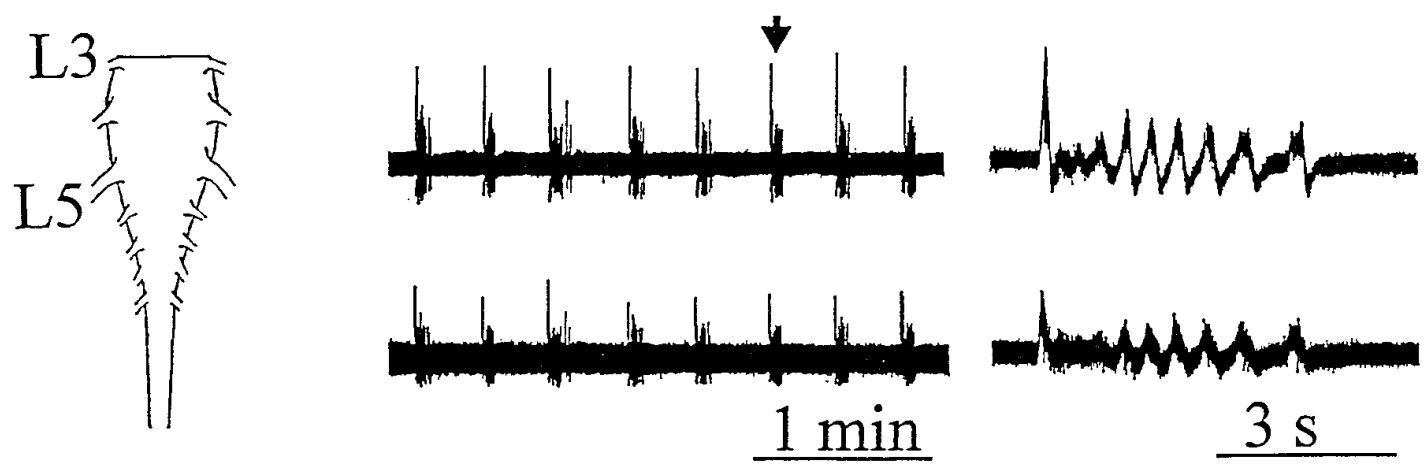

B
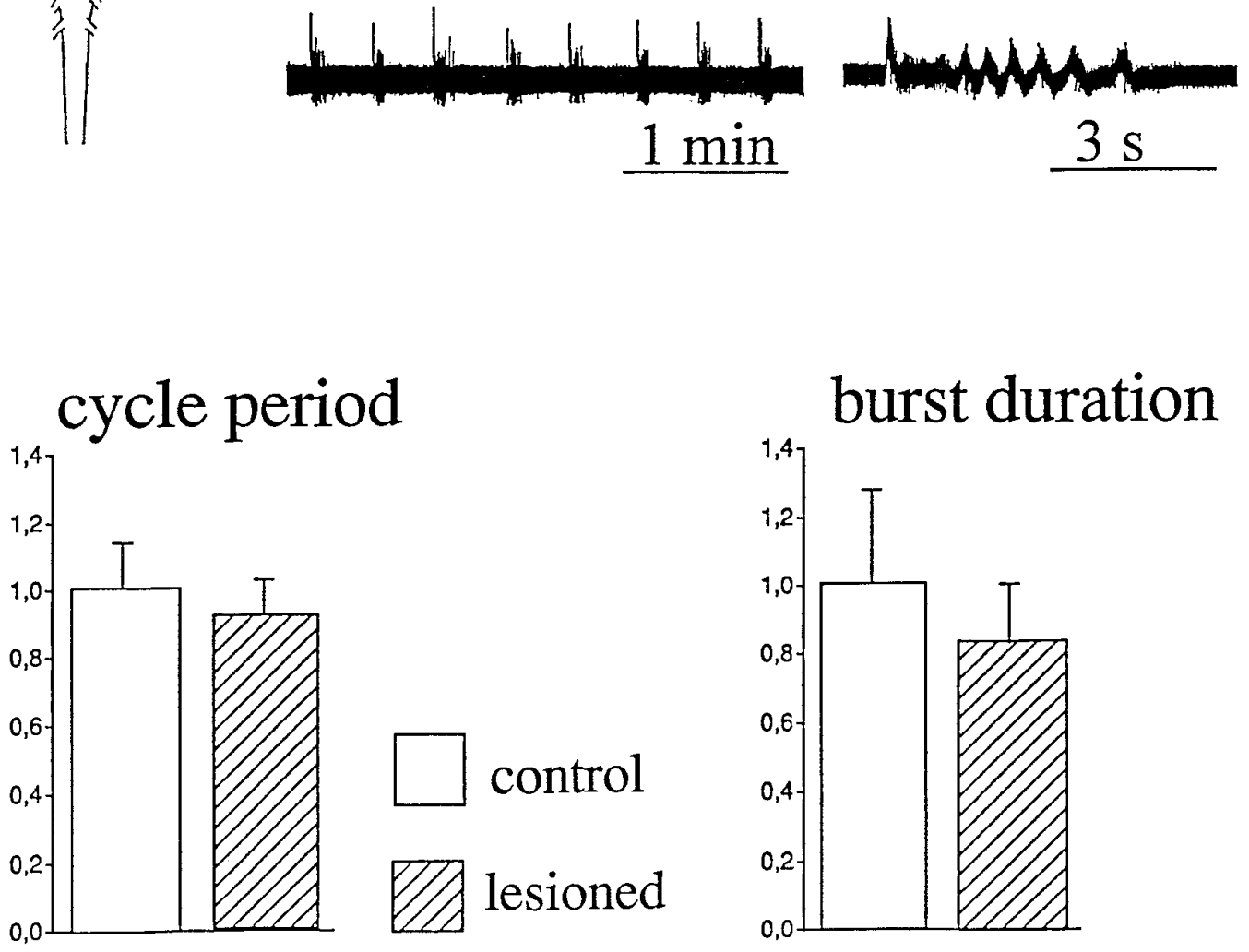

Figure 4. Effect of cross-sectioning the spinal cord at L3 level on bursting activity. A, AC-coupled sample records of bursting induced by strychnine and bicuculline (see legend to Fig. 3 for further details; no $20 \mathrm{~Hz}$ filtering) in an isolated preparation (intact) and after removal of the cord region rostral to L3 (see schematic diagram on the left). Right traces are faster records of arrowed events. B, Histograms of cycle period (left) or burst duration (right) calculated from six preparations before (open columns) or after (hatched columns) this type of lesion. In this and all subsequent figures (unless otherwise indicated), data are normalized with respect to control values before the lesion. 
shown by the histograms from 6 preparations (Fig. $4 B$, right). It seemed also of interest to explore the minimum number of segments necessary to support bursting. In four cases, the portion of the cord localized caudal to L6 was subsequently removed without change in burst frequency and duration (data not shown). Furthermore, a tissue block containing only L4 and L5 segments ( $n=$ 3) generated bursts (recorded from L5 ventral root) of a more irregular nature, as indicated by their elevated coefficient of variation for the cycle period (on average 75 vs $12 \%$ in the intact cords). Burst duration was on average decreased by $59 \%$ with respect to the intact preparations. A block of tissue comprising the L5 segment only $(n=3)$ displayed irregular and infrequent spontaneous bursts occurring on average at $7.3 \pm 3.7$ min intervals and lacking intraburst oscillations (burst duration $3.3 \pm 2.6 \mathrm{sec}$ ).

\section{Sagittal sectioning of the spinal cord}

Because the present pattern consists of simultaneous bursting of homologous ventral roots, we tested whether side-to-side neural connections at different segmental levels were necessary to maintain synchronicity. To this end, we first performed progressive hemisectioning from the caudal part of the cord (as indicated by dotted lines at the top of Fig. $5 A$ ) while recording from left and right $\mathrm{L} 5$ ventral roots. Figure $5 A$ shows that, even when the cord was bisected up to L4 or L2 (termed type I or II lesion, respectively), rhythmic bursts still took place simultaneously in L5 ventral roots. Similar results were obtained in four preparations. In the example of Figure $5 A$, sagittal hemisectioning also produced shortening of individual burst duration. This phenomenon, however, was not statistically significant when data from four preparations were pooled together (see below). The average values observed for burst frequency and burst duration are illustrated in the plots of Figure $6 A$, in which the type I and II lesions are grouped as caudal hemisection protocol (open columns). Although burst frequency and burst duration were not significantly affected by these lesions, the coefficient of variation of the cycle period (but not the one of burst duration) was largely increased $(+168 \%)$ after type II lesion. Thus, such a lesion affected the regularity of burst onset without any concomitant change in burst duration regularity. When the cord was further split up to L1 level, complete synchronicity was maintained in two of four preparations. Further splitting up to thoracic level resulted in burst desynchronization in all cases (data not shown).

Analogous results were observed when the hemisection was performed starting from the rostral end of the cord (rostral hemisection protocol; $n=4$ ). In this case, bursts still took place simultaneously in L5 ventral roots even when the cord was split down to L6 (type III lesion) or S2 (type IV lesion), as shown in Figure $5 B$. The effects of these lesions on the average values of cycle period and burst duration are plotted in Figure $6 B$. Even in this case, no significant changes were observed in these values, but the coefficient of variation of the cycle period was largely increased after lesions III and IV ( +531 and $+590 \%$, respectively). Moreover, burst duration appeared to decrease after each lesion, although this effect was not statistically significant. Further splitting of the spinal cord resulted in partial or complete loss of synchronicity in all cases. Thus, side-to-side connections localized more than three segments apart from L5 level (either rostrally or caudally) are sufficient to maintain rhythmic bursts synchronous in L5 ventral roots.

Complete hemisection of the spinal cord was performed on four intact preparations. In all cases, rhythmic bursting in left and right L5 ventral roots persisted without synchronicity. In two cases the two halves displayed different burst frequency and duration (see Fig. 5C), whereas in the other two cases burst frequency and duration were similar for the left and right sides, although no longer temporally locked together. Plots of Figure $6 C$ (obtained by pooling all data from both sides of each preparation) show that, in addition to a strong increase in the coefficient of variation of cycle period, complete hemisection caused a significant reduction in burst duration. These data demonstrate that each side of the spinal cord possessed the neural substrate necessary to produce rhythmic activity in the presence of strychnine and bicuculline.

\section{Effects of dorsal horns ablation}

After dorsal horn removal from the chick embryo spinal cord, the remaining network, localized in the ventral horns, is still able to perform coordinated rhythmic activity (Ho and O'Donovan, 1993). To test whether in the neonatal rat spinal cord ventral horn networks were able to generate rhythmic activity in the absence of synaptic inhibition, we recorded from L5 ventral roots before and after a frontal section that removed the dorsal horns and the central canal area from spinal cord preparations comprising lumbosacral segments. In five such preparations, histological examination of stained sections confirmed that only the laminae localized ventrally to the central canal had been left (see example in Fig. $7 B$ ). In all of these cases, rhythmic bursts were still observed in lesioned preparations, as illustrated by a representative example in Figure $7 A$. Whereas the cycle period was unaffected by dorsal horn removal, a significant reduction in burst duration was consistently observed even if intraburst oscillations were still detectable. Average values of cycle period and burst duration before and after dorsal horns ablation are plotted in Figure $7 C$.

\section{Rhythmic activity in isolated ventral horns}

In four preparations exposed to strychnine and bicuculline, rhythmic bursting was studied after surgical removal of dorsal horns plus one ventral horn that left an isolated spinal cord quadrant. Such a lesion, performed on a preparation consisting of four to five lumbosacral segments, was confirmed histologically on transverse slices of the tissue (see example in Fig. $8 \mathrm{~B}$ ). Figure $8 \mathrm{~A}$ illustrates recordings from L5 ventral root before and after this procedure. Rhythmic bursts were still present after lesion; their frequency was larger than in control, whereas their duration decreased. Similar results were observed in the other preparations. The average values for cycle period and burst duration are plotted in Figure $8 C$. In all cases, burst frequency increased after this lesion, with a concomitant decrease in burst duration. Both effects were statistically significant (Fig. $8 C$ ). The oscillatory structure of bursts, typical of intact preparations, was always lost after this lesion, and bursts were converted from multicomponent events (lasting on average $\sim 7 \mathrm{sec}$ ) to shorter $(1-3 \mathrm{sec})$ single discharges (Fig. 8A).

\section{Sensitivity of lesioned preparations to 5-HT and NMDA}

In fully hemisected spinal cords and in preparations consisting of isolated ventral horns, application of 5-HT $(10 \mu \mathrm{M})$ or NMDA (5 $\mu \mathrm{M})$ in the presence of strychnine and bicuculline always resulted in a concomitant increase of burst frequency and decrease of burst duration, similar to that observed in intact spinal cord [see Fig. 3 and related text for NMDA effects; see also Bracci et al. (1996) for 5 -HT effects on intact preparations]. In the presence of 5-HT, cycle period and burst duration were significantly $(p<0.0001)$ decreased by $40 \pm 11$ and $53 \pm 15 \%$, respectively, in hemisected cords $(n=3)$ and by $59 \pm 10$ and $37 \pm 9 \%$, respectively, in dorsal horn-ablated preparations $(n=3)$. In the presence of NMDA, 


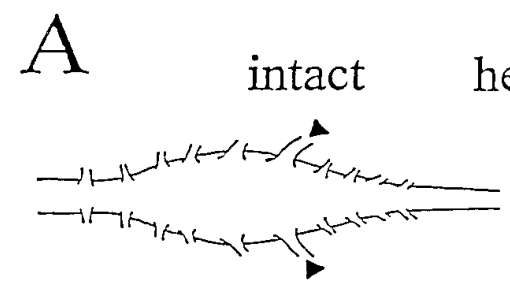

\section{hemisection caudal to L4}
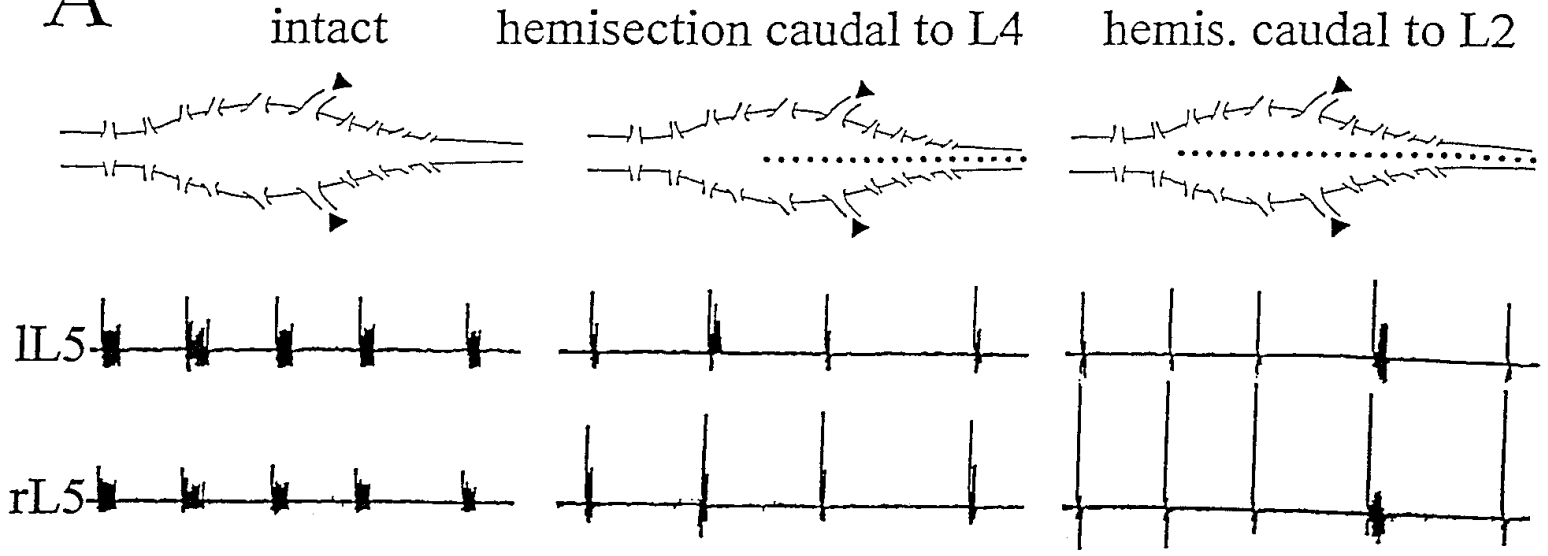

$\mathrm{B}$
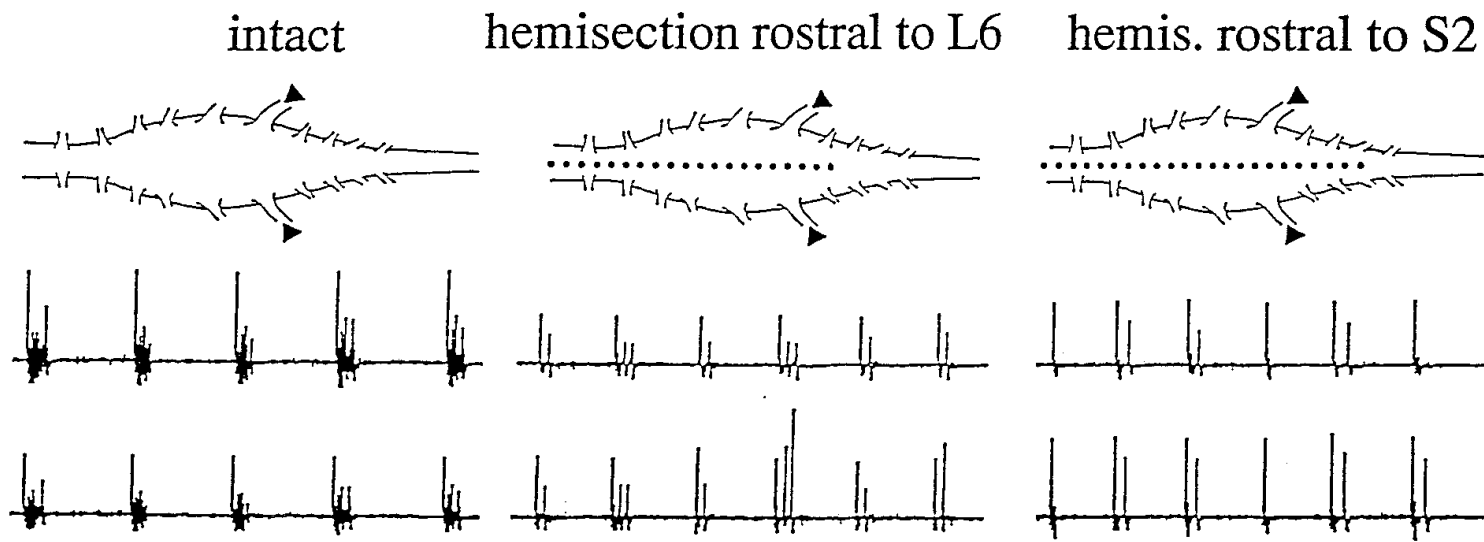

$\mathrm{C}$
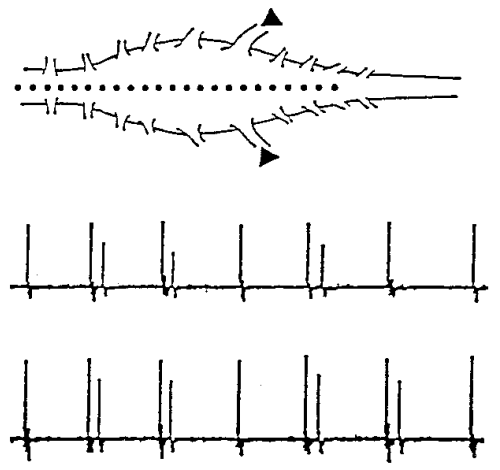

$1 \mathrm{~min}$
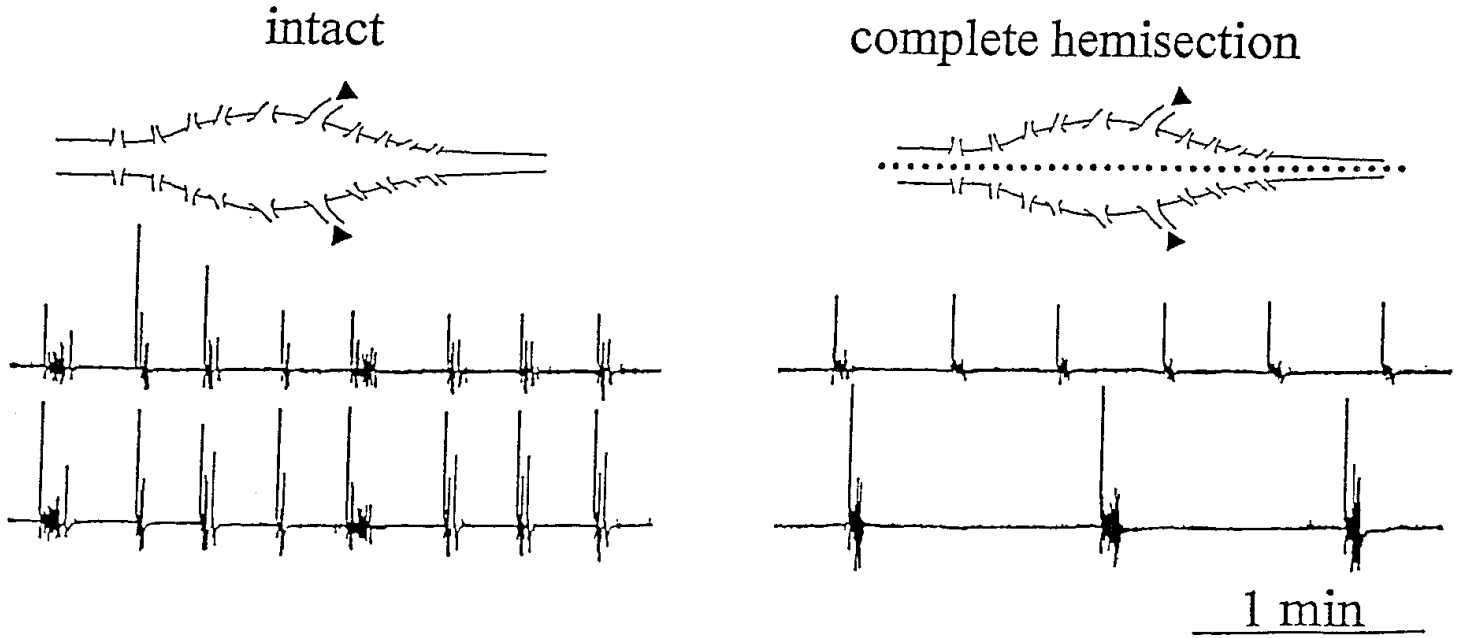

Figure 5. Effects of sagittal hemisectioning of the spinal cord on bursting activity. A, AC-coupled tracings (from left and right L5 ventral roots marked by filled triangles; $20 \mathrm{~Hz}$ lowpass filtering) of bursting activity from the isolated preparation (intact; left) and after two types of lesion bisecting the cord from the caudal end up to L4 (middle) or to L2 (right). Schemes of these lesions are shown above corresponding traces. Synchronized bursting (with shorter burst duration) persists after these lesions. $B$. Similar tracings from a preparation in which the bisectioning was started from the rostral end down to L6 (middle) or S2 (right) as indicated by schemes above traces. Also, in this case bilateral synchronicity of L5 bursting is preserved. $C$, Example of bursting activity from a preparation before (left) and after (right) complete hemisectioning of the spinal cord. The latter procedure leads to alteration in the frequency of bursting for each root, loss of synchronicity between the left and right ventral roots, and shorter burst duration. 

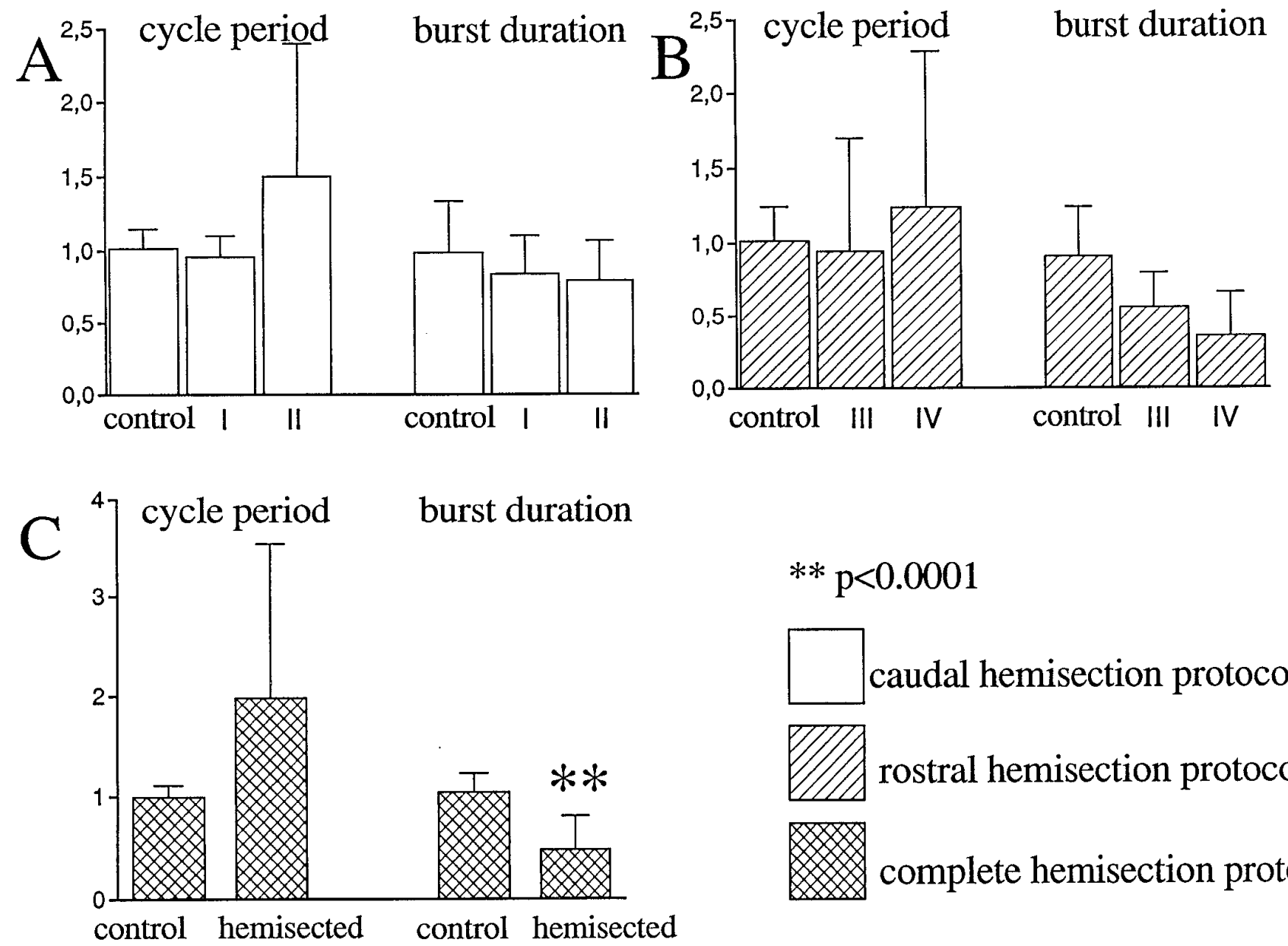

$* * \mathrm{p}<0.0001$
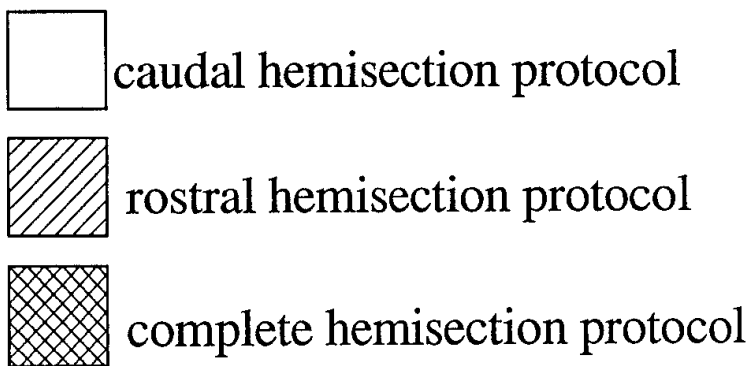

Figure 6. Histograms of changes in cycle period and burst duration after partial or complete hemisection of the spinal cord. $A-C$ represent three lesion protocols, namely, hemisectioning from the caudal end (open columns), from the rostral end (hatched columns), or complete hemisection (cross-hatched columns). Data are averages from four preparations for each protocol. The extent of hemisectioning in $A$ and $B$ is indicated as $I, I I, I I I$, or $I V$ (see Results for further details): note that when hemisectioning splits a considerable length of the cord (type $I I$ or $I V$ lesions), there is an increase in the SD of the cycle period (reflecting increased variability of burst frequency) even if average values do not differ significantly. Complete hemisectioning $(C)$ largely increases the variability of the cycle periods and significantly $\left({ }^{* *} p<0.0001\right)$ reduces burst duration.

cycle period and burst duration were significantly $(p<0.0001)$ decreased by $45 \pm 16$ and $56 \pm 10 \%$, respectively, in hemisected cords $(n=2)$ and by $62 \pm 9$ and $43 \pm 13 \%$, respectively, in dorsal horn-ablated preparations $(n=3)$. In isolated ventral quadrants (in which bursts were reduced to single-discharge events), only cycle period was significantly $(p<0.0001)$ decreased by NMDA or 5 -HT $(62 \pm 21$ and $55 \pm 18 \%$, respectively; $n=3)$. In these preparations, however, burst duration was not significantly affected by these agents. This situation is illustrated in the example of Figure $9 A$ : in the presence of strychnine and bicuculline alone, regular rhythmic bursts were recorded from L5 ventral root of an isolated ventral horn. These events were $<2 \mathrm{sec}$ in duration and lacked intraburst oscillations, which were replaced by a plateau with sustained action potential firing (as indicated by thickening of tracing during burst; right panels of Fig. 9A). Application of $5 \mu \mathrm{M}$ NMDA produced a strong increase in burst frequency $(310 \%)$ but failed to affect burst duration significantly (see plots of Fig. 9B). The faster tracings of individual bursts shown in the right panels of Figure $9 A$ also indicate that there was no alteration in intraburst structure in the presence of NMDA. These results suggest that two different mechanisms could underlie burst triggering and burst structure. The second mechanism was probably lost after surgical isolation of a single ventral quadrant, resulting in brief and unstructured bursts, while the mechanism responsible for burst onset was still present. As a consequence, further pharmacological manipulations with 5-HT or NMDA were able to affect burst triggering only.

\section{DISCUSSION}

The principal finding of this study is that spontaneous rhythmic bursts induced in the neonatal rat spinal cord by strychnine and bicuculline were present in the following spinal structures even after their surgical isolation from the rest of the cord: (1) spinal segments caudal to L3; (2) longitudinally hemisected spinal cord; (3) isolated ventral horn preparations; and (4) single ventral quadrant.

\section{Block of synaptic inhibition}

Because many models of spinal rhythmogenesis rely on synaptic inhibition (Grillner et al., 1991; Dale, 1995; Roberts et al., 1995), it was important to investigate whether inhibition was actually suppressed during the present rhythm. Although on neonatal rat motoneurons strychnine and bicuculline are known to block $\mathrm{GABA}_{\mathrm{A}}$ and glycine receptors, respectively (Wu et al., 1992), the 

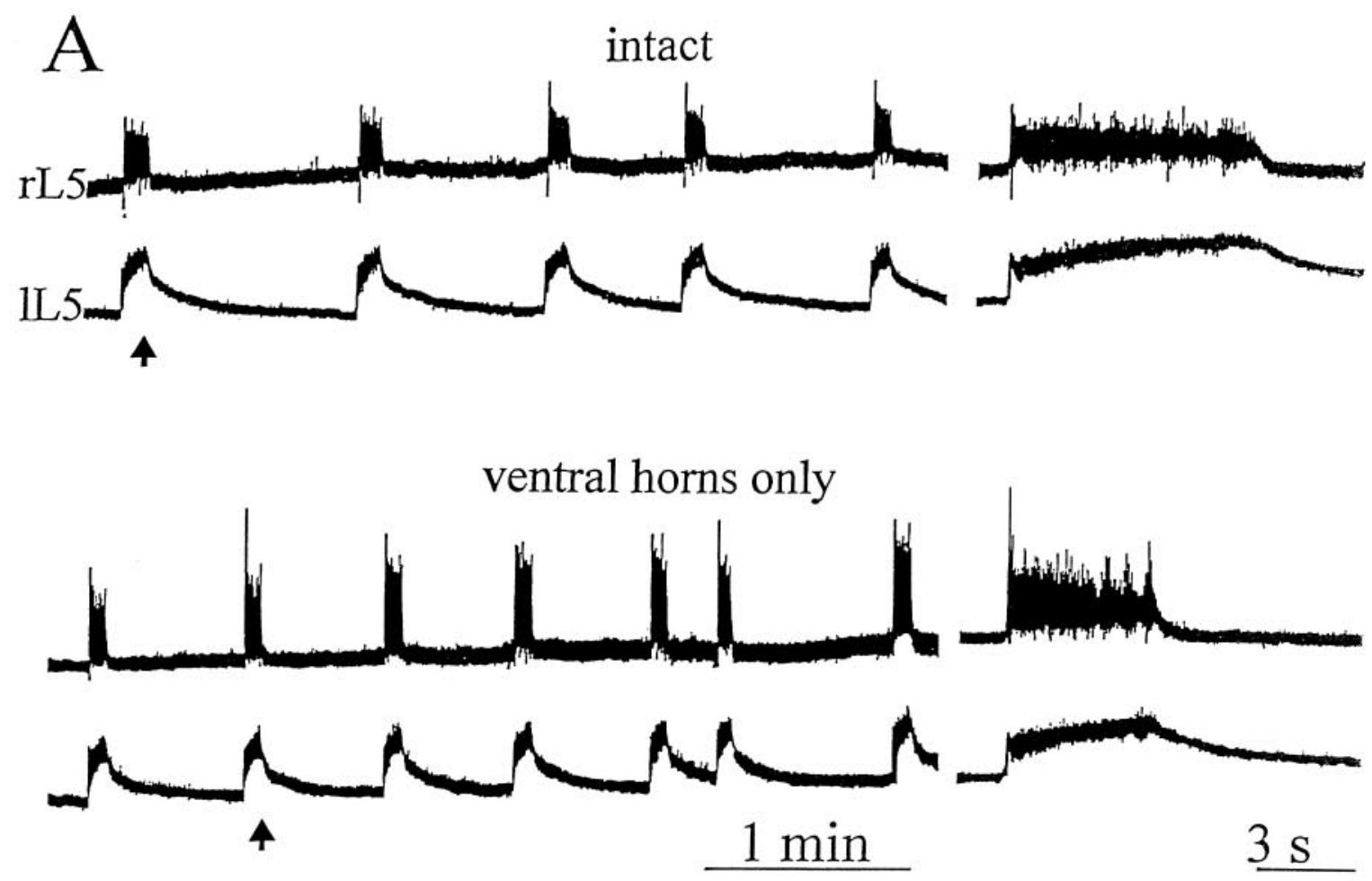

$\mathrm{B}$
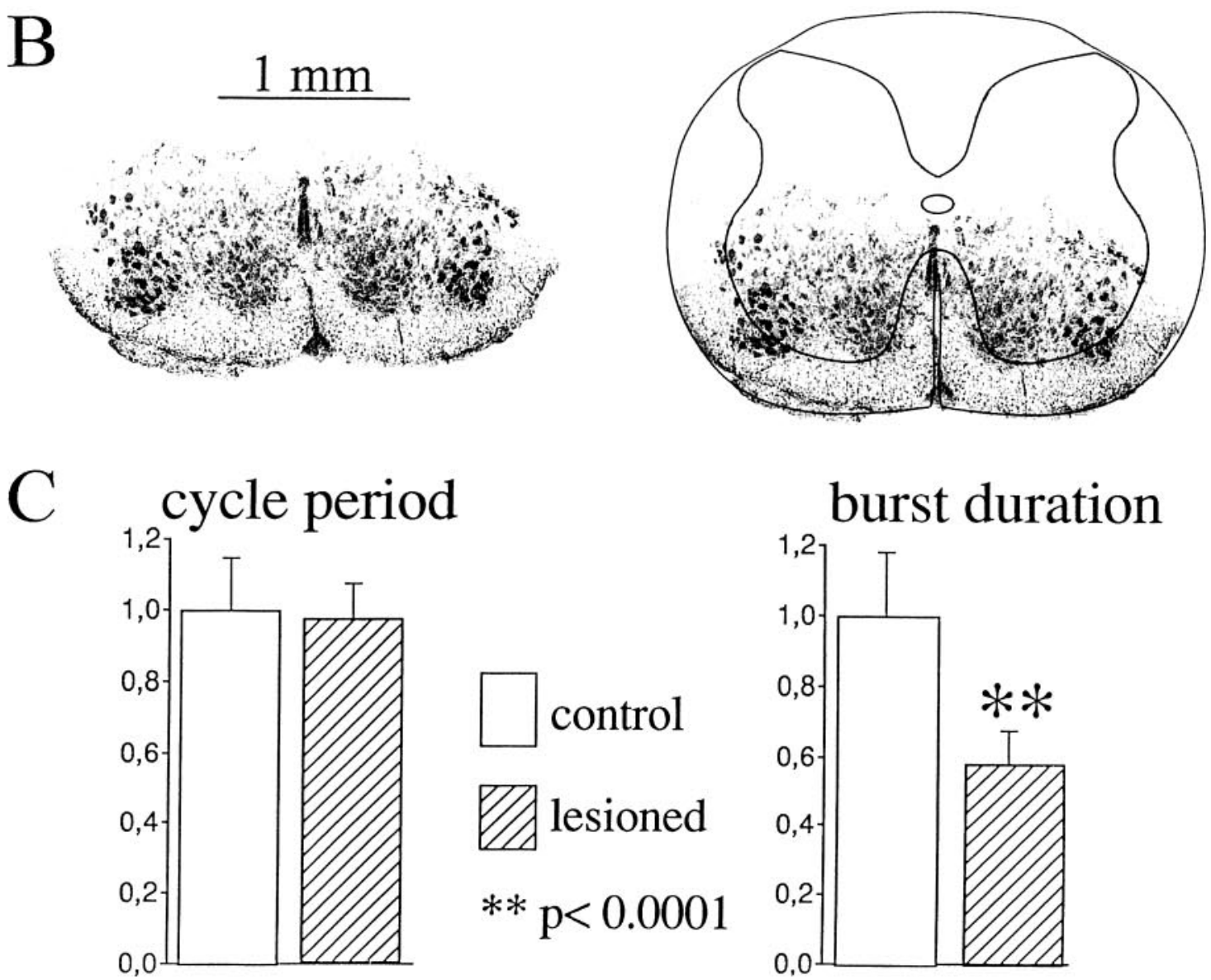

Figure 7. Effect of ablation of dorsal horns on bursting activity. $A$, DC-coupled records of bursting activity from L5 ventral roots before (intact; top) and after frontal section (ventral horns only; bottom), which removes dorsal horns and area below midline (see stained section of this preparation in $B$, either alone or within the schematic contours of the spinal cord). Right traces are shown with a faster time base to depict individual burst (arrows) time course. Note that this procedure shortens burst duration. C, Histograms of normalized cycle periods (left) and burst duration (right) for unlesioned (open columns) or lesioned (hatched columns) preparations $(n=4)$. Burst duration is significantly decreased $(* * p<0.0001)$. 


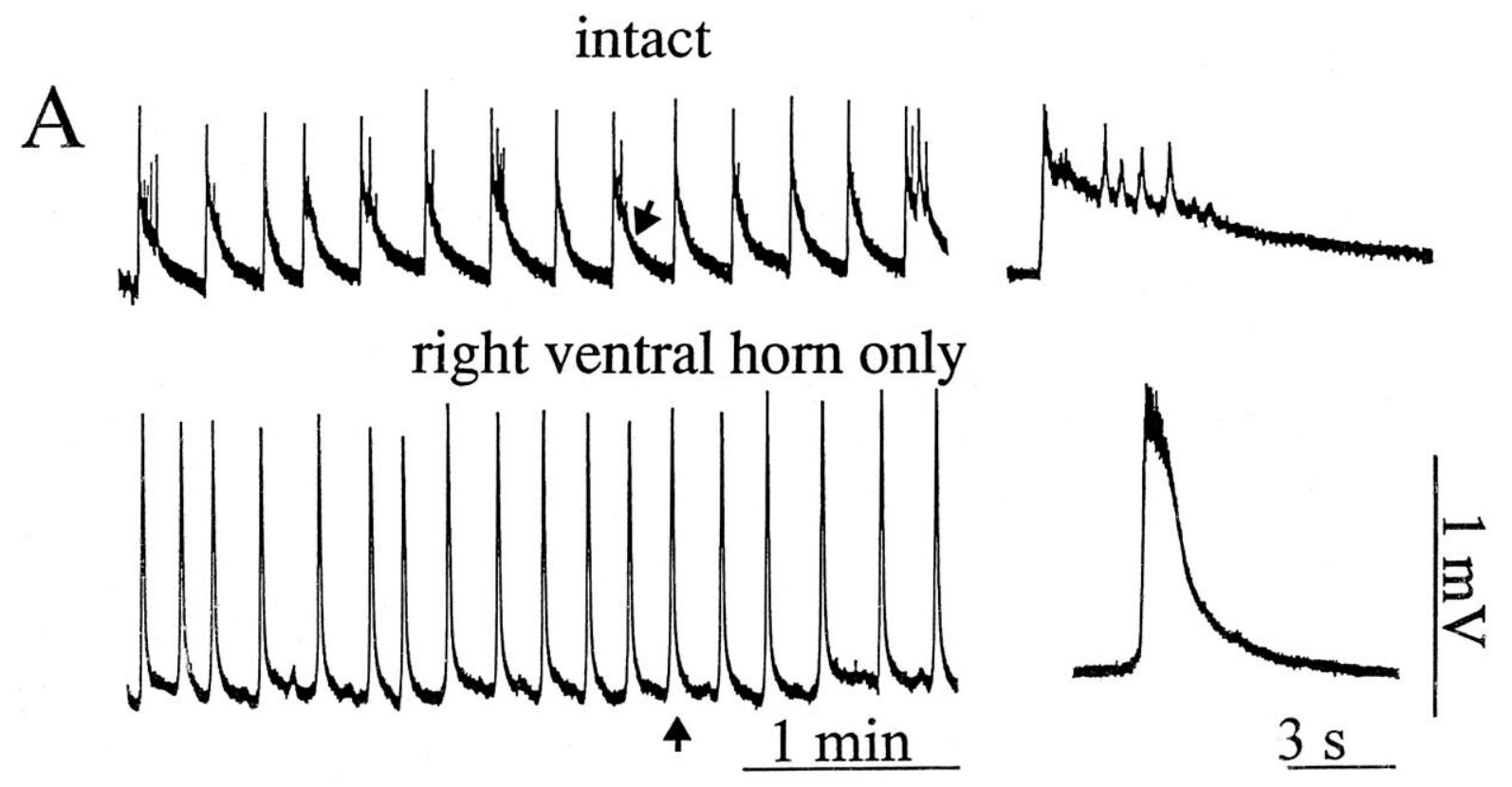

B
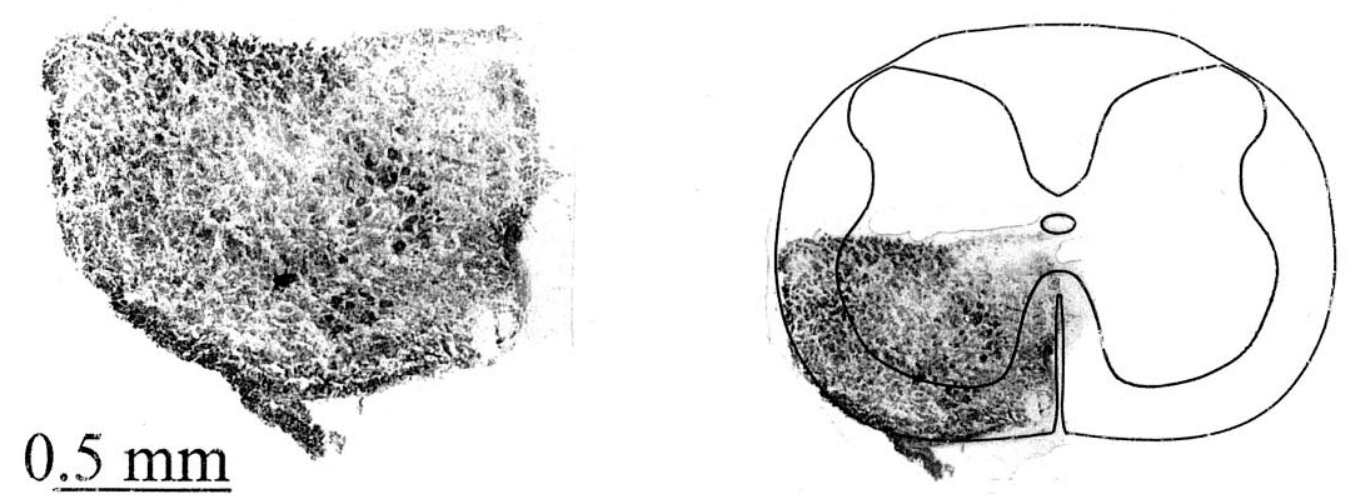

C cycle period
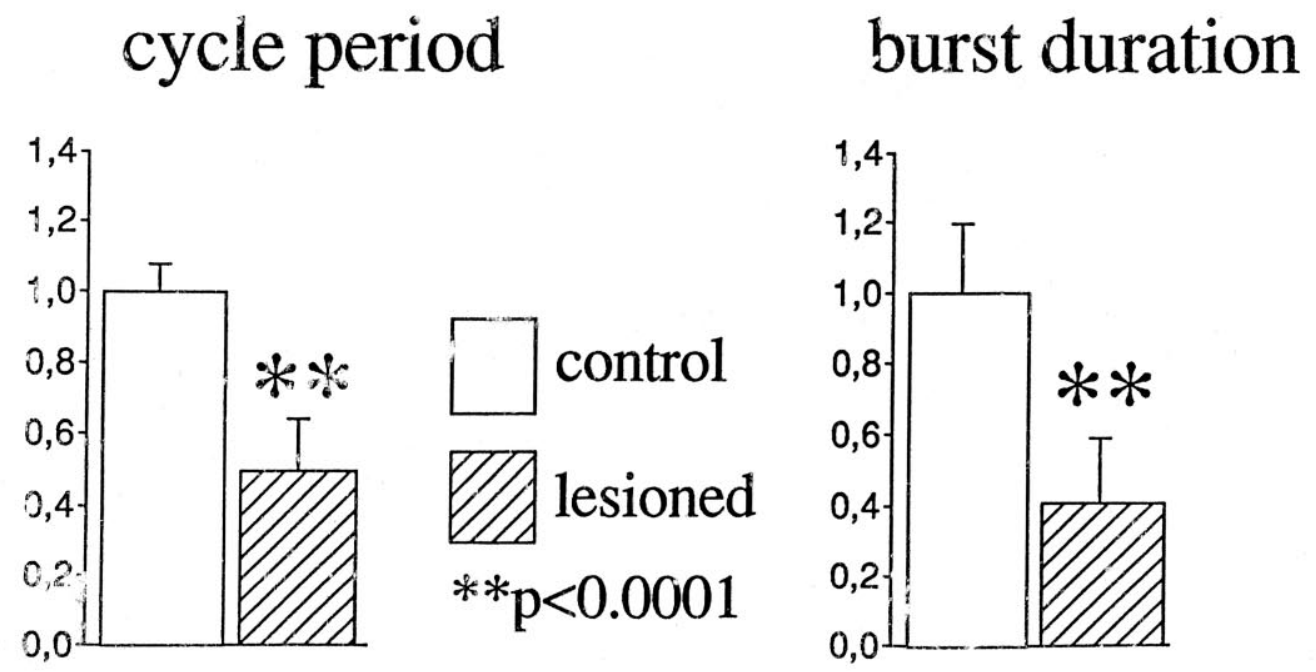

Figure 8. Bursting activity in an isolated ventral horn quadrant. $A$, DC-coupled tracings (from right L5 ventral root) in isolated preparation (intact; top) and after surgical isolation (bottom) of a ventral quadrant. The latter is shown in $B$ histologically either alone or within the idealized contours of the spinal cord. Responses indicated by arrows are shown on the right side of $A$ on a faster time base. $C$, Histograms of cycle period (left) or burst duration (right) for unlesioned (open columns) or lesioned (hatched columns) preparations $(n=4)$. ${ }^{* *} p<0.0001$ 
A

strychnine and bicuculline

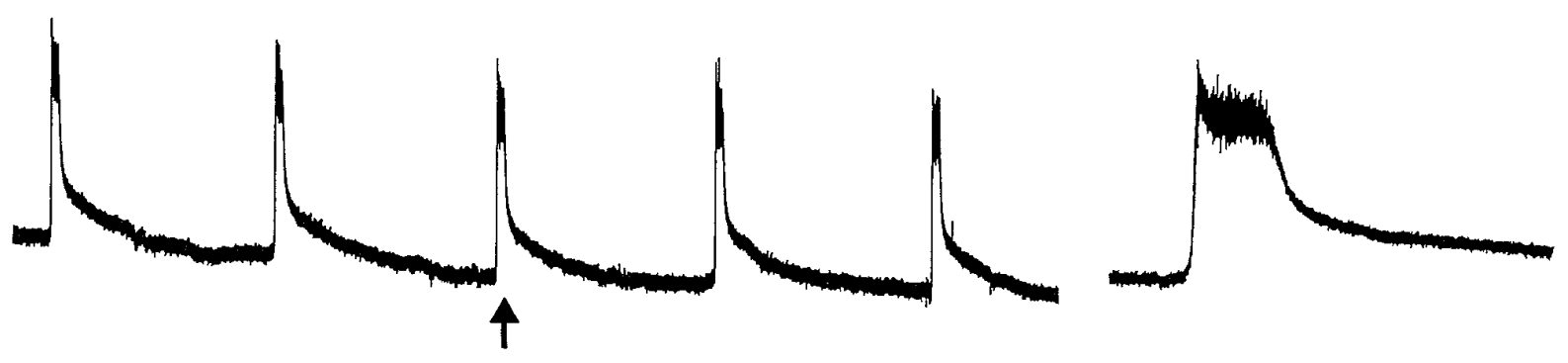

\section{$+5 \mu \mathrm{M}$ NMDA}

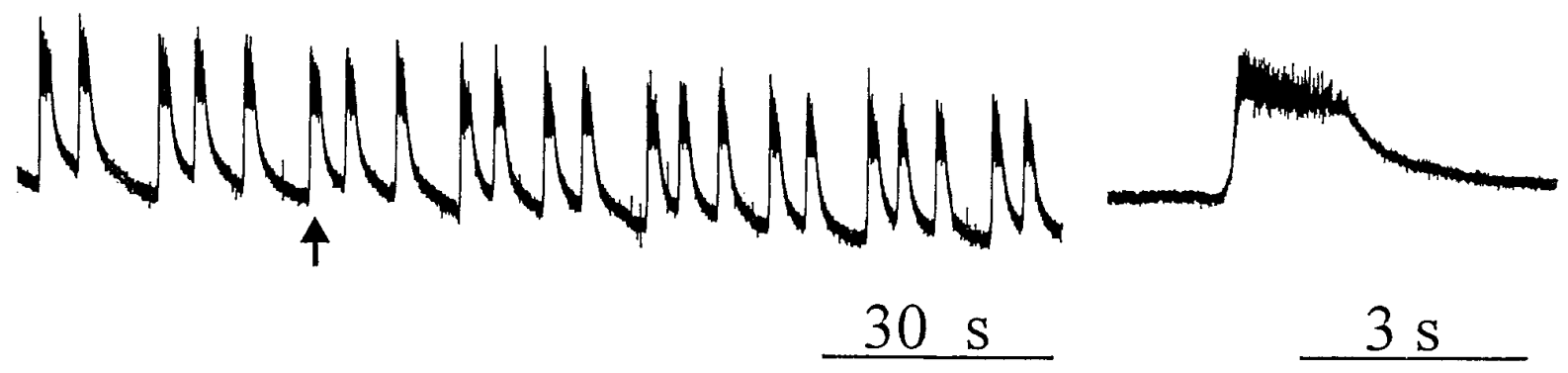

B

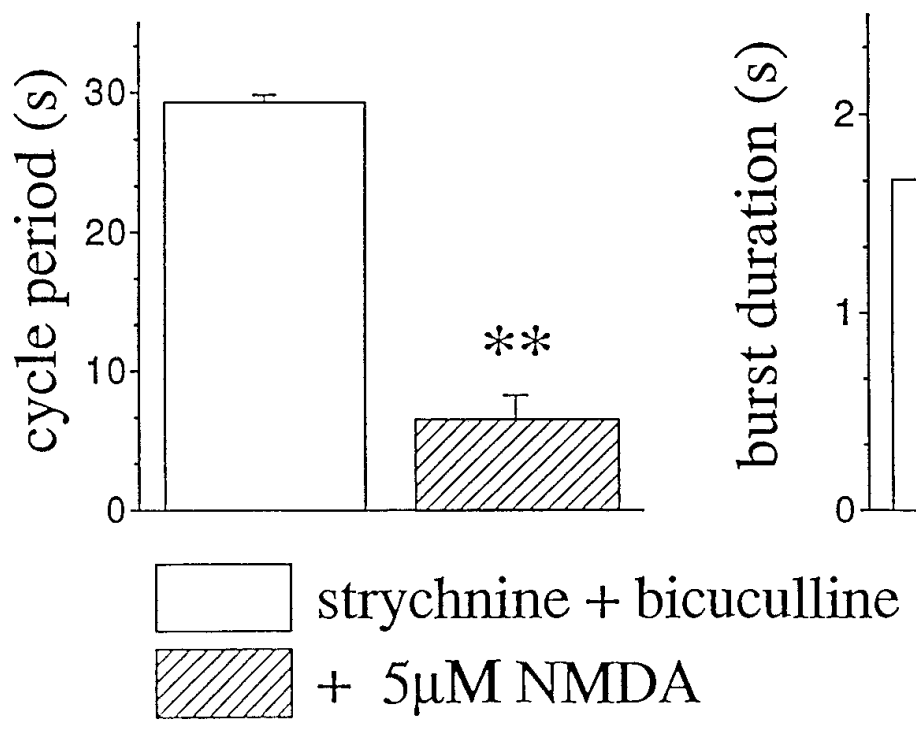

Figure 9. Sensitivity of bursting to NMDA in a ventral quadrant preparation. $A$, DC-coupled tracings of bursting activity from left L5 ventral root in a ventral quadrant preparation (for details, see legend to Fig. 8) in strychnine and bicuculline solution (top) to which NMDA (5 $\mu \mathrm{M})$ is then added (bottom). Right traces are shown with faster time base. Note increase in burst frequency in the presence of NMDA. $B$, Histograms of cycle period (in sec; left) or burst duration (right) in strychnine + bicuculline solution (open columns) or after addition of NMDA (hatched columns). Data are means of values observed during 5 min periods. ${ }^{* *} p<0.0001$. 
present experiments were undertaken to validate this notion throughout the spinal cord networks. GABA (in the presence of CGP 52,432) or glycine failed to affect strychnine- and bicucullineinduced bursts. Furthermore, when the strychnine concentration was progressively elevated from 1 to $10 \mu \mathrm{M}$, no additional change in bursting pattern became apparent, thus suggesting that $1 \mu \mathrm{M}$ strychnine had already saturated glycine receptors in accordance with the high affinity of this antagonist (Becker et al., 1988). These data indicate that inhibitory receptors were effectively blocked by the two alkaloids. The noncompetitive $\mathrm{GABA}_{\mathrm{A}}$ antagonists picrotoxin or penicillin produced effects that were indistinguishable from the ones caused by bicuculline, suggesting that bicuculline actually exerted its action through block of $\mathrm{GABA}_{\mathrm{A}}$ receptors. Because CGP 52,432 did not block bursts, it is also apparent that $\mathrm{GABA}_{\mathrm{B}}$ receptor activation was not required for such a rhythm.

Whereas strychnine or bicuculline largely increased dorsal rootevoked reflex activity, only strychnine caused the appearance of 4-8 Hz time-locked neuronal oscillations. These oscillations are similar to the ones intrinsic to the spontaneous bursts in strychnine and bicuculline solution and, because they were absent during the irregular bursting elicited by bicuculline alone (Bracci et al., 1996), presumably imply a particular role for glycinergic inhibition in the decay phase of polysynaptic excitatory responses. Similarly, Cowley and Schmidt (1995) have shown that application of strychnine (but not of bicuculline) during fictive locomotion evokes $4-8 \mathrm{~Hz}$ packets of synchronized intraburst discharges. Thus, it seems likely that these oscillations specifically arose from block of glycinergic inhibition.

\section{Local networks supporting rhythmic bursting}

Lesions of the neonatal rat spinal cord showed the following. (1) Removal of segments rostral to L3 had no effects on burst frequency and duration, although more than two segments were necessary to preserve regular rhythmicity. (2) Gradual splitting of the spinal cord from the caudal end increased the variability of burst frequency but not of burst duration. (3) Gradual splitting from the rostral end had similar effects on burst variability and tended to reduce burst duration. (4) Bilateral synchronicity between the activity of the two L5 ventral roots persisted even when crossed connections remained intact only at a distance of several segments (either rostrally or caudally). (5) After complete separation of the spinal cord into left and right hemicords, both sides continued to independently perform rhythmic bursting at different rates, even if this lesion increased burst frequency variability and decreased burst duration. (6) Ablation of the tissue dorsal to the central canal did not affect burst frequency but resulted in reduction of burst duration. (7) Although the characteristic structure of bursts with several oscillations was usually retained after the above-mentioned lesions, in the case of the ventral quadrant preparation bursts were converted into simple single-discharge events at higher frequency. Thus, it became apparent that the minimal neuronal network necessary for this rhythmic activity was contained within a single ventral quadrant spanning over a few lumbar segments.

On isolated spinal cords or lesioned preparations in which intraburst oscillations were still present, 5-HT or NMDA always produced a concomitant decrease in cycle period and burst duration. Conversely, in the case of isolated ventral quadrants these drugs enhanced burst frequency without changing burst duration. This finding suggests that the rhythmic onset of bursts and their prolonged oscillations were due to two different mechanisms. In support of this view, Streit (1996) observed that, on organotypic cultures of the rat spinal cord, lesions could selectively affect either duration or frequency of bursting induced by block of synaptic inhibition. In the present study, each type of lesion left the mechanism responsible for rhythmic burst onset intact, while the mechanism responsible for burst oscillations was impaired by hemisection and dorsal horns ablation and was lost after ventral quadrant isolation. It seems likely, therefore, that burst oscillations depended on the number of cells left in the network and that such a number was insufficient in the case of a single ventral quadrant, which was able to generate rhythmicity but incapable of supporting long duration bursts.

The cellular and network mechanisms supporting the observed bursting activity are still to be clarified. Because bursting appeared in the absence of synaptic inhibition and was not crucially dependent on NMDA receptor activity (see Bracci et al., 1996), the present results cannot be explained by models used for other rhythmogenic patterns such as fictive locomotion, which are based on reciprocal inhibition and NMDA receptor-driven pacemaker neurons (for review, see Grillner et al., 1995). In the present case, one can speculate that bursts originated via an autoregenerative mechanism (relying on AMPA receptor activity) (Ballerini et al., 1995; Bracci et al., 1996) within a network comprising excitatory connections only and were terminated perhaps by a combination of synaptic depression (Streit, 1993) and activation/inactivation of voltage-dependent conductances of premotoneurons (Bracci et al., 1996).

\section{Comparison with other investigations}

The relation between the present bursting pattern, observed in the absence of synaptic inhibition, and fictive locomotion (induced by 5-HT and/or NMDA) has yet to be clarified. In this sense, three possibilities can be envisioned. (1) These patterns are produced by two different and separated spinal networks whose behavior is unrelated. (2) The pattern observed in the presence of strychnine and bicuculline is produced by networks structurally overlapping the ones involved in fictive locomotion, but these patterns are produced by different cellular mechanisms. (3) These patterns are attributable to the same basic rhythm generator, and the differences in terms of spatiotemporal phasing are attributable to additional networks, comprising inhibitory processes, that ensure alternation in the presence of synaptic inhibition. Although further investigations are required to discriminate between these hypotheses, it is important to note that indirect support to the third hypothesis arises from the fact that agents traditionally used to elicit fictive locomotion (the frequency of which depends on drug concentration; Cazelets et al., 1992) were also able to accelerate the pattern induced by strychnine and bicuculline in a similar dose-dependent manner. Cazalets et al. (1995) have proposed that in the neonatal rat spinal cord fictive locomotion is produced by a rhythm-generating network localized in the rostral lumbar region at L1-L2 level. Such a rostrolumbar localization is compatible only with the first hypothesis because the rhythmgenerator processes responsible for the pattern in the absence of inhibition remained after removal of segments rostral to L3. However, in the chick embryo spinal cord, Ho and O'Donovan (1993) have shown that even the caudal lumbosacral segments can perform rhythmic bursting similar to fictive locomotion, although they are less rhythmogenic than the rostral segments. These results, if applicable to the rat spinal cord, would allow using hypotheses two and three to explain the present data. In particular, within the framework of the third hypothesis, the rat caudal segments might possess locomotor rhythm generators that are unmasked by strychnine and bicuculline more easily than by 5-HT and NMDA. 
In the Xenopus embryo spinal cord, rhythmic activity persists after the left and right sides have been separated (Kahn and Roberts, 1982) or in the presence of saturating concentrations of strychnine (Soffe, 1989); numerical models based on reciprocal inhibition between left and right pools hardly account for these findings (Dale, 1995). Thus, it seems likely that, as in the case of the neonatal rat, even in the Xenopus embryo spinal cord there is a rhythmogenic mechanism that does not require synaptic inhibition for its operation.

Rhythmic activity has been observed in other CNS preparations during block of synaptic inhibition. Brainstem respiratory networks in vitro have been shown to produce spontaneous rhythmic activity whose frequency is not changed by block of chloride-mediated synaptic inhibition (Feldman and Smith, 1989). In hippocampal slices, block of $\mathrm{GABA}_{\mathrm{A}}$ receptor results in rhythmic bursts characterized by a primary discharge followed by several oscillations (Miles et al., 1984; Traub et al., 1993; Taylor et al., 1995). In this case, two mechanisms have been proposed to be responsible for the primary discharge (attributed to activation of AMPA receptors at recurrent excitatory synapses) and for late oscillations (attributed to dendritic calcium spikes; Traub et al., 1993).

\section{Concluding remarks}

Because block of synaptic inhibition biases synaptic processes toward excitation in the spinal cord, this pharmacological tool provides a large experimental simplification of the neuronal circuitry to investigate the properties of the spinal networks. The findings that the collective behavior of spinal neurons, far from becoming chaotic under this conditions, turns into an extremely regular pattern, which can be strongly modulated by several chemical agents and is preserved after ablation of large portions of neuronal tissue, represent an important constraint to be included in spinal network models.

\section{REFERENCES}

Becker CM, Hoch W, Betz H (1988) Glycine receptor heterogeneity in rat spinal cord during postnatal development. EMBO J 7:3717-3726.

Ballerini L, Bracci E, Nistri A (1995) Desensitization of AMPA receptors limits the amplitude of EPSPs and the excitability of motoneurons of the rat isolated spinal cord. Eur J Neurosci 7:1229-1234.

Bracci E, Ballerini L, Nistri A (1996) Spontaneous rhythmic bursts induced by pharmacological block of inhibition in lumbar motoneurons of the neonatal rat spinal cord. J Neurophysiol 75:640-647.

Cazalets JR, Sqalli-Houssaini Y, Clarac F (1992) Activation of the central pattern generators for locomotion by serotonin and excitatory amino acids in neonatal rat. J Physiol (Lond) 455:187-204.

Cazalets JR, Borde M, Clarac F (1995) Localization and organization of the central pattern generator for hindlimb locomotion in newborn rat. J Neurosci 15:4943-4951.
Cowley KC, Schmidt BJ (1994) A Comparison of motor pattern induced by $N$-methyl-D-aspartate, acetylcholine and serotonin in the in vitro neonatal rat spinal cord. Neurosci Lett 171:147-150.

Cowley KC, Schmidt BJ (1995) Effects of inhibitory amino acid antagonists on reciprocal inhibitory interactions during rhythmic motor activity in the in vitro neonatal rat spinal cord. J Neurophysiol 74:1109-1117.

Dale N (1995) Experimentally derived model for the locomotor pattern generator in the Xenopus embryo. J Physiol (Lond) 489:489-510.

Feldman JL, Smith JC (1989) Cellular mechanisms underlying modulation of breathing pattern in mammals. Ann NY Acad Sci 437:114-130.

Fisher ND, Nistri A (1993) A study of the barium-sensitive and -insensitive components of the action of thyrotropin-releasing hormone on lumbar motoneurons of the rat isolated spinal cord. Eur J Neurosci 5:1360-1369.

Grillner S, Wallen P, Brodin L (1991) Neuronal network generating locomotor behaviour in lamprey. Annu Rev Neurosci 14:169-199.

Grillner S, Deliagina T, Ekeberge O, El Manira R, Hill H, Lansner A, Orlovsky N, Wallen P (1995) Neural networks that co-ordinate locomotion and body orientation in lamprey. Trends Neurosci 18:270-279.

Ho S, O'Donovan MJ (1993) Regionalization and intersegmental coordination of rhythm-generating networks in the spinal cord of the chick embryo. J Neurosci 13:1354-1371.

Hultborn H, Kiehn O (1992) Neuromodulation of vertebrate motor neuron membrane properties. Curr Opin Neurobiol 2:770-775.

Kahn JA, Roberts A (1982) The central nervous origin of the swimming motor pattern in embryos of Xenopus laevis. J Exp Biol 99:185-196.

Katz PS (1996) Neurons, networks and motor behavior. Neuron 16:245-253.

Lev-Tov A, Pinco M (1992) In vitro studies of prolonged synaptic depression in the neonatal spinal cord. J Physiol (Lond) 447:149-169.

Miles R, Wong RKS, Traub RD (1984) Synchronized afterdischarges in the hippocampus: contribution of local synaptic interaction. Neuroscience 12:1179-1189.

Roberts A, Tunstall MJ, Wolf E (1995) Properties of networks controlling locomotion and significance of voltage dependency of NMDA channels: simulation study of rhythm generation sustained by positive feedback. J Neurophysiol 73:485-495.

Rossignol S, Dubuc R (1994) Spinal pattern generation. Curr Opin Neurobiol 4:894-902.

Soffe SR (1989) Roles of glycinergic inhibition and $N$-methyl-D-aspartate receptor mediated excitation in the locomotor rhythmicity of one half of the Xenopus embryo central nervous system. Eur J Neurosci 1:561-571.

Streit J (1993) Regular oscillation of synaptic activity in spinal networks in vitro. J Neurophysiol 70:871-878.

Streit J (1996) Mechanisms of pattern generation in cocultures of embryonic spinal cord and skeletal muscle. Int J Dev Neurosci 14:137-148.

Taylor GW, Merlin LR, Wong RKS (1995) Synchronized oscillation in hippocampal CA3 neurons induced by metabotropic glutamate receptor activation. J Neurosci 15:8039-8052.

Traub RD, Miles R, Jefferys JGR (1993) Synaptic and intrinsic conductances shape picrotoxin-induced synchronized after-discharges in the guinea-pig hippocampal slice. J Physiol (Lond) 461:525-547.

Wu W, Ziskind-Conhaim L, Sweet MA (1992) Early development of glycine- and GABA-mediated synapses in rat spinal cord. J Neurosci 12:3935-3945. 